\title{
Diffuse colonic lipomatosis presenting as acute abdomen with intraoperative resemblance of colonic carcinoma and concealed perforation
}

\author{
Rusiru Kalhara Ranaweera, A. N. K. Abayajeewa \\ National hospital of Sri Lanka
}

\begin{abstract}
Keywords: Colonic lipomatosis; carcinoma colon; concealed perforation

\section{Introduction}

Diffuse colonic lipomatosis is a rare condition characterized by the presence of lobules of adipose tissue in the submucosa of the whole colon affected[1]. Though rare, colonic lipomatosis can present with features of obstruction, perforation and intussusception[2]. They can also mimic colonic carcinoma intraoperatively[3]. There have been reports of five cases of diffuse colonic lipomatosis in literature before[2][3]. Herein we report the sixth case of diffuse colonic lipomatosis resembling colonic carcinoma and concealed perforation.
\end{abstract}

\section{Case presentation}

A 49-year-old female patient presented with intermittent abdominal pain for the one-month duration with pain increasing in severity for 2 days duration. She also complained of abdominal distention and vomiting. She has not opened her bowel for 2 days but was passing flatus. There was no history of fever. She was a diagnosed patient with chronic pancreatitis. On examination, there was severe tenderness and guarding of the right lower abdomen. The initial WBC count was $12.22 \times 109, \mathrm{Hb}$ was $9.5 \mathrm{~g} / \mathrm{dl}$ and S.amylase was $105 \mathrm{U} / \mathrm{L}$. Initial ultrasound scan of abdomen suggested inflammatory bowel mass in the right iliac fossa region with suspicion of an appendicular mass. Contrastenhanced CT abdomen and pelvis showed a thickened terminal ileum with mural oedema of the ascending colon. The inflamed terminal ileal loops were seemed to form an inflammatory mass which made the bowel loops difficult to be traced. There were multiple air pockets within the mass and it was difficult to comment whether they were intraluminal or extraluminal. The appearance was suggestive of "terminal ileitis forming into an infective mass."

The patient underwent an exploratory laparotomy. There was blood stained peritoneal fluid entering into the peritoneal

Correspondence: Rusiru Kalhara Ranaweera

E-mail: kalhara.ranaweera@gmail.com

(i) https://orcid.org/0000-0002-1433-7072

Received: 31-07-2021 Accepted: 07-11-2021 DOI: http://doi.org/10.4038/sljs.v39i3.8859 cavity. There was firm to hard irregular mass extending from the distal ileum, caecum to the proximal part of ascending colon. There was no mesenteric lymph node enlargement. On the caecum, there was suspicion of a concealed perforation. Right hemicolectomy was done with side to side anastomosis of distal ileum to the proximal transverse colon. Postoperatively patient recovered uneventfully.

In the histopathology report, gross examination revealed adhered ileum, caecum with appendix and a segment of colon with the hemorrhagic and congested serosal surface. The caecum and terminal ileum were dilated. There was an area with a suspicion of a rupture through the serosa of the caecum. The cut surface showed a thick yellow tissue throughout the submucosa with overlying flattened mucosa. The serosa of the appendix appeared inflamed. There was no diverticula, tumour polyps or caseous necrosis on extensive sectioning.

On microscopic examination, sections of the colon showed intact but diffusely flattened mucosa. The submucosa showed extensive infiltration of mature fat cells causing thinning of the muscularis propria. The serosa showed dilated, congested blood vessels and a moderate amount of inflammatory cell infiltrate. The mucosa of the caecum and ileum showed ulceration with a heavy mixture of inflammatory cell infiltrate, extending from mucosa to serosa, which showed many dilated congested blood vessels. The appendix showed normal histology except for moderate serosal inflammation.

There was no evidence of granulomata or malignancy. Terminal ileum and ascending colon up to transverse colon features were compatible with diffuse colonic lipomatosis with serositis involving the ileum, caecum and colon with evidence of perforation in the caecum adjacent to the appendix.

\section{Discussion}

Lipomas of the colon are the second most common benign tumour of the colon after adenomas[3]. Incidence is high in the fifth and sixth decades of life with a slight female preponderance[5]. Colonic lipomas are commonly submucosal [90\%], the rest being serosal [4]. Lipomas are usually single[3]. In colonic lipomatosis, multiple benign lipomas are present throughout the colon. If lobules of adipose 


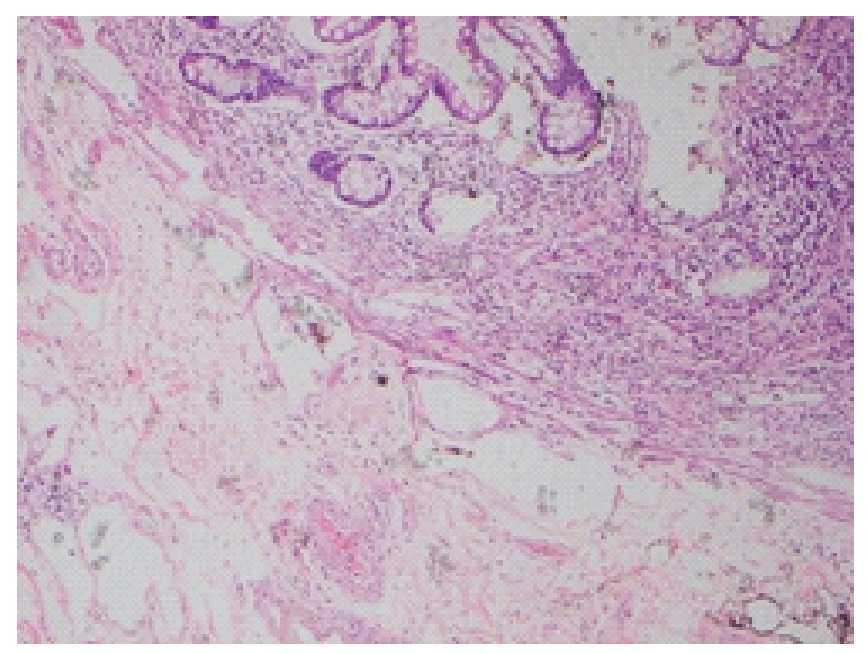

Figure 1. Infiltration of fat in the submucosa

tissue are present only in one segment of the colon it is known as segmental colonic lipomatosis and if lobules of adipose tissue affect the whole colon it's known as diffuse colonic lipomatosis [3].

The majority of patients with colonic lipomatosis are asymptomatic. In most patients, colonic lipomas are detected incidentally during investigation for symptoms associated with the gastrointestinal system. On rare occasions, patients can present with obstruction, perforation or intussusception[1].

Another important clinical significance is that it can resemble carcinoma colon. In this patient gross appearance intraoperatively was similar to carcinoma colon with a concealed perforation.

Colonic lipomatosis can be diagnosed by histopathology. CT abdomen, Barium studies may be helpful but they are not conclusive of the disease.
Common indications for surgical intervention in colonic lipomatosis are the complications associated with it, such as intestinal obstruction, intussusception, perforation and bleeding.

\section{Conclusion}

Colonic lipomatosis is a very rare occurrence in clinical practice. Sometimes it can present as acute abdomen. Intraoperatively on gross examination, it can resemble carcinoma colon.

All authors disclose no conflict of interest. The study was conducted in accordance with the ethical standards of the relevant institutional or national ethics committee and the Helsinki Declaration of 1975, as revised in 2000 .

\section{References}

1. Ramirez JM, Ortego J, Deus J, Bustamante E, Lozano R, Dominguez M. Lipomatous polyposis of the colon. Br J Surg [Internet]. 1993 Mar [cited 2020 Feb 22];80[3]:349-50. Available from: http://www.ncbi.nlm.nih.gov/pubmed/8472149. https://doi.org/10.1002/bjs.1800800329

2. Snyder CW, Cannon JA, Snyder CW, Cannon JA. Diffuse Intestinal Lipomatosis Presenting as Adult Intussusception Diffuse Intestinal Lipomatosis Presenting as Adult Intussusception. 2013;3[1].

3. Sandhu PS, Bansiwal RK, Attri AK, Mittal R. Diffuse Colonic Lipomatosis, Presenting as Perforation Peritonitis and Mimicking Carcinoma Colon. Indian J Surg. 2011 Apr;73[2]:155-7.

https://doi.org/10.1007/s12262-010-0204-3

4. González-Urquijo M, Kettenhofen SE, Rodarte-Shade M. Colonic intussusception by a giant colon lipoma: A case report. Int J Surg Open. 2017;9:7-9.

https://doi.org/10.1016/j.ijso.2017.08.001

\section{Learning Points:}

- Colonic lipomatosis is a rare condition that can present as acute abdomen.

- Intraoperatively findings of colonic lipomatosis can be confused with carcinoma colon. 